MACHUCO ROSA, Antonio

Faculdade de Letras da Universidade do Porto (Portugal)

@ machuco.antonio@gmail.com

\title{
Mimesis, network theory and digital social networks
}

\section{Mimesis, teoría de redes y redes sociales digitales}

We analyse the formal theory of networks and the new digital media from the perspective of mimetic theory. It is emphasised that the spatial form of the new media is characterised by distribution functions that are power laws. We show that this spatial form can be derived from the presence of mimetic desire in digital social networks. Finally, we show that network theory allows the representation of the scapegoat mechanism as it was analysed by René Girard.

KEY WORDS: Mimesis, network theory, digital social networks.

Analizamos la teoría formal de las redes y los nuevos medios digitales desde la perspectiva de la teoría mimética. Se enfatiza que la forma espacial de los nuevos medios se caracteriza por funciones de distribución que son leyes de poder. Demostramos que esta forma espacial puede derivarse de la presencia del deseo mimético en las redes sociales digitales. Finalmente, mostramos que la teoría de redes permite la representación del mecanismo de chivo expiatorio tal como fue analizado por René Girard.

PALABRAS CLAVE: Mimesis, teoría de redes, redes sociales digitales.

\section{INTRODUCTION}

It is becoming increasingly clear that the work of René Girard has allowed new ways of looking at the foundations of various areas of the social sciences, from anthropology to economics, political science and psychology. How mimetic theory can be developed with the support of formal models derived from mathematics and physics has been less discussed, probably because Girard himself never expressed interest in that subject in his published writings. There are, however, some authors who have shown that this perspective is perfectly possible. The best example is perhaps provided by the work of Jean-Pierre Dupuy. From the eighties of the last century, he sought to call attention to the pertinence of the then emergent studies of complex systems for Girard's theory. ${ }^{[1]}$ At the same time, André Orléan (1985) proposed a formal model of the scapegoat mechanism, a point that our article also addresses. 
A little more recently, Lucien Scubla (1996) used the so-called «catastrophe theory» to formalise Girard's theory of sacrifice. There are some other examples, but the foregoing are enough to show that Girard's work is deeply resonant with the scientific developments that occurred in parallel with the elaboration of his theory.

On the other hand, there have been even fewer studies analysing the recently created digital social networks from the perspective of mimetic theory. As Geoff Shullenberger (2016) has pointed out, «that omission is surprising,» and it has probably occurred because Girard himself had nothing to say about technology in his published oeuvre. However, a quick look at features such as «like», «follower» and «share» on Facebook or Instagram show that they are related to imitation and allow us to anticipate that mimetic theory can effectively be an important tool to understand the role that digital social networks play today. As will be mentioned below, René Girard's ideas even led one of his former students to make millions from an initial investment in Facebook.

So, there are two aspects that Girard's theory and its later developments have, at least in part, neglected: on the one hand, the importance of formal models describing complex systems, and on the other, how new networks based on the Internet, such as the World Wide Web and the interactive platforms Facebook or Instagram, grow through the mechanisms of imitation and positive feedback. In this article, we propose the integration of these two topics within the framework of mimetic theory. More specifically, we will use a particular type of complex formal system: a network. The modern formal theory of networks is a central core in this article, and it is also the theory that allows a systematic study of digital social networks' interactive platforms. In both topics, we will show the importance of a general principle of imitation, and in particular, the importance of mimetic desire.

In the first section of the article, we present the elementary concepts of the mathematical theory of networks. Next, we note that some recent empirical results show that the original theory of networks must be reformulated in order to account for interactions grounded on imitation. This will be done on the basis of a formal model proposed by Albert Barabási. It will be underlined that the distribution function of the links between the nodes of a network has a very particular form: a mathematical distribution with the shape of a power law. In the fourth section, we will provide a phenomenological description of how mimetic desire is present in the new digital platforms, showing that they can be analysed with the help of formal network theory. In the following section, we present what we think is the main result of this article. Based on an empirical and theoretical study, it will be shown that the spatial form of the new digital networked media, i.e. power law distributions, can be deduced from mimetic desire. Mimetic desire is at the origin of the spatial structure of the new digital social networks! Finally, in the last section, we will return to the formalisation of imitation in the framework of network theory to show that, under certain circumstances, the theory can describe, at an elementary level, the central mechanism proposed by Girard's theory, i.e. the mechanism of the convergence of all individuals towards a single individual, that is, the scapegoat mechanism.

\section{NetWORK THEORY}

Modern network theory was created in the middle of the last century at a time when formal models of social phenomena largely neglected the interactions between the agents and 
behaviours represented in those models. Network theory describes the properties of a type of topological space that can be constructed through a mathematical model in which the agents are independent of each other and their connections are random. According to the model, a network can be defined as follows. ${ }^{[2]}$

- A network is a set of nodes, $n$, connected by links (also called edges);

- A node has a degree $k$ (the number of links in each node).

The following figure illustrates some very simple networks, showing the case where there is a cycle, or triangle, in a network.

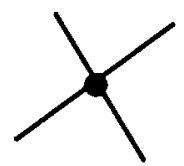

$\mathrm{K}=4$

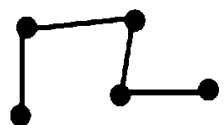

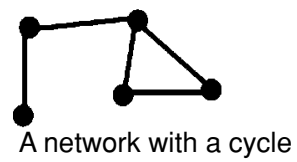

Figure 1. Some examples of networks.

Based on this elementary definition, the first formal model of networks was proposed by Paul Erdós (1959). It begins with a number of nodes that are successively connected. More precisely, the model starts with $n$ nodes and no links, and then each pair of nodes is randomly connected with probability $p$. This probability can be calculated as the fraction between the existing links and all the links that may possibly exist on the network (these are equal to $n$ $(n-1 / 2))$. An example is shown in Figure 2.

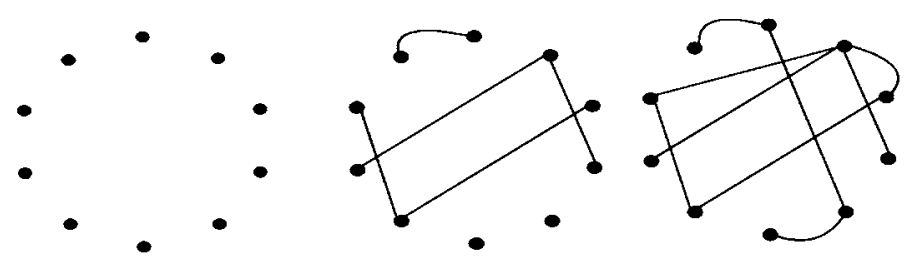

Figure 2. The Erdó's model. We start with $n$ nodes, and then each pair of nodes is randomly connected with probability $p$. From left to right, we have $p=0, p \approx 0,1, p=0,2$ of the $n(n-1) / 2$ possible links in the network. At $p \approx 0,1$ a giant cluster emerges.

An important aspect of this type of construction is the existence of critical phase transitions. This means that certain properties emerge at a critical point. If a certain property emerges at a critical point, this means that the emergence takes place on a very rapid temporal scale when compared to the time scale of the whole process of network construction. An important emergent property is when a «giant» cluster appears at a sudden moment in the construction of a random network. A «giant cluster» emerges when the parameter on which the network construction depends exceeds a certain critical value. In the case of random networks, this parameter is the probability, $p$, of two nodes being connected. It can be shown (Bollábas, 1985 ) that there is a critical probability $p_{c}$ with critical value $p \approx 1$, that is, when $p \approx 1 / n$, a giant 
cluster emerges (see again Figure 2). Of course, one speaks of a «giant cluster» in relation to the size of the network. The size of the giant cluster becomes «almost» the size of the whole network in the asymptotic boundary of $n$, where it is possible to reach almost any node from any other. The emergence of a giant cluster is an important property of networks, and it should be mentioned that a formal process equivalent to the emergence of a giant cluster in random networks has been proposed for decades as a way of formalising the basic mechanism (the unanimity of opinions of all against one individual) of René Girard's theory (Orléan, 1985). Based on the theory of networks, at the end of this article, we will propose an alternative mechanism to the one proposed by André Orléan.

For the moment, rather than analysing the existence of critical transitions in the Erdós model, it is more important to mention another fundamental property of networks that we consistently insist on throughout this article. It is the distribution function, $P(k)$, of the links between the nodes. It is relatively intuitive that in a network in which there is a fixed number of nodes, and the connections between the nodes are created randomly, the distribution function is a normal function (a Poisson or Gaussian distribution), that is, any node has, on average, the same number of links as any other node (see figure 3 ).

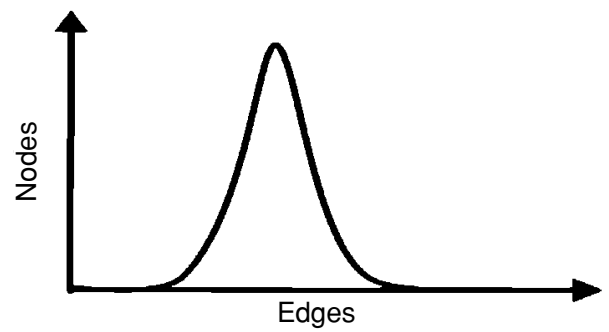

Figure 3. A normal distribution: $k$ is on average the same in each node.

Due to the construction process of the Erdós model, it was expected that the final distribution would be a normal one. It should indeed be noted that this type of network has no memory, i.e. each new link is independent of the existing links. There is really no interaction between the elements (the nodes) of the system.

Although they are not relevant properties in the context of this article, it can be mentioned that, besides the existence of a giant cluster and the distribution function, there are other quantities that are investigated in network theory. One is the distance, which is the average number of links that exist between any two nodes in the network. Another, the clustering coefficient, allows an analysis of the existence of cycles in the network, that is, the situation in which if a node $A$ is connected to $B$ and $B$ to $C$, then $A$ is also connected to $C .^{[3]}$ It is a property that allows the detection of the existence of communities and the clustering of opinions in a network, but we will not address those topics here.

\section{The World Wide Web}

For decades, it was thought that the Erdós model could be a plausible description of real networks. In fact, it is only a mathematical construction, and it is now known that in the vast 
majority of empirical networks, the nodes are not randomly connected. Above all, the distribution function of real networks is very far from being a normal distribution. This became clear when, at the end of the last century, an Internet-based network, the World Wide Web (WWW), was systematically investigated. This network was created by Tim Berners Lee around 1991. His revolutionary idea was to build a new universal media of communication; a universal networked media. These two properties are now well known: The World Wide Web supports any format of information (universality), and it is really a network, that is, a set of nodes (web pages) linked or not linked to each other by hyperlinks. Contrary to expectations at the time, Albert Barabási and collaborators (Albert et al 1999) verified that the distribution function, $P(k)$, of the $k$ hyperlinks between the nodes (web pages) has the form $P(k) \sim k^{\lambda}$, i.e. a distribution without characteristic scale, or a power law. In general terms, this means that the probability of a randomly chosen node (page) to receive $k$ links decreases according to the ratio given by the exponent $\lambda$. The following figure illustrates a power law.

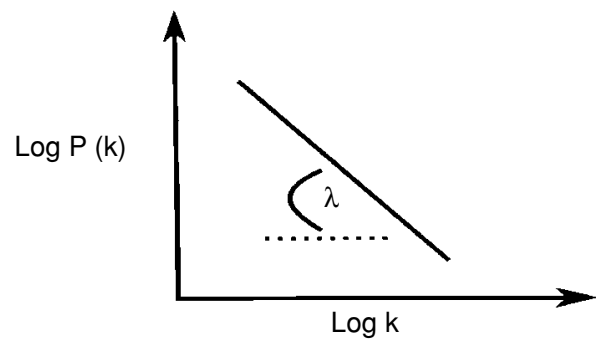

Figure 4. A power law. The probability of a randomly chosen node having degree $k$ decays like a power of $k$, where the exponent $\lambda$, typically measured in the range $2<\lambda<3$, determines the rate of decay.

In intuitive terms, that distribution means that there are few pages that receive a large number of links, and a large number of pages that receive few links. That is, the majority of nodes have less-than-average degree, and a small fraction of «big» nodes (hubs) are many times more connected than the average. It is a distribution that is rather different from a normal (Gaussian) distribution, in which, on average, the number of links is the same on all pages. An important point about power law distributions is indeed that the number of links decreases according to the constant ratio given by the exponent $\lambda$. See the following Figure 5 , which compares a network with a normal distribution versus one without a characteristic scale. Nodes with the highest number of connections (hubs) are shown in grey.
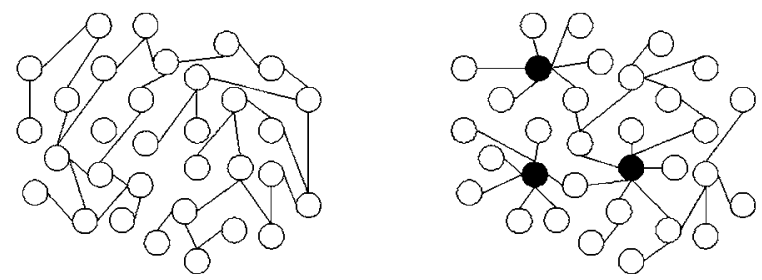

Figure 5. On the left, a random network with a normal distribution. On the right, a power-law distribution network. Hubs are shown in grey. 
The initial study by Albert et al. (1999) conducted on the nd.edu domain of the WWW found $\lambda \approx 2.1$. It is a value similar to another study on the overall WWW (Broder et al., 2000). Here, we present the results of Broder et al. (cf. Figure 6), whether for incoming hyperlinks (pages directed from hyperlinks) or for outgoing hyperlinks (pages that have hyperlinks to other pages). It is an important distinction, since the WWW is an oriented network, i.e. if there is a hyperlink between pages $A$ and $B$, it does not necessarily follow that there is also one from $B$ to $A$ ).
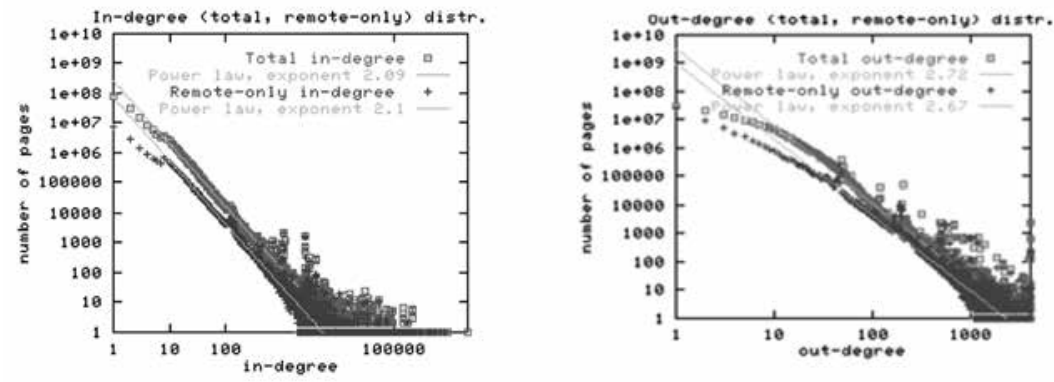

Figure 6. In and Out distribution functions obtained from a World Wide Web search. Source: Broder et al. (2000). Value of 2.1 for the case of inlinks and 2.7. for the case of outlinks.

We can define the popularity or attention that a page gets by the existence of hyperlinks that point to them, so that pages are very popular (have a large number of incoming links) by virtue of users creating many hyperlinks (outgoing links) that point to these popular pages. As can be seem in Figure 6, outgoing links also exhibit a power law. The WWW structure is simultaneously created by nodes (pages) that point to other pages and by nodes pointed to by other pages. It should be noted that the value (2.7) of the exponent in the case of outlinks is clearly higher than the value (2.1) in the case of inlinks. In both cases, there is a power law, but there is a pronounced asymmetry between the value of the two distributions. In the case of inlinks distribution, there is an even smaller number of nodes that captures a disproportionately large number of links. More precisely, $5 \%$ of nodes receive $75 \%$ of all links (incoming links), while they only account for $35 \%$ of all links pointing to other pages. As we will show below, this difference tends to be abolished in more recent networks, showing that there is a tendency towards undifferentiation in the evolution of digital networked media.

A system that follows a power law is a self-organised structure that emerges spontaneously from various local acts creating pages and links, and therefore, is not the result of an intentional design. These acts cause the system to evolve towards a stationary critical state. That critical state has the signature of a power law, which means that there is not a normal scale: A powerlaw distribution is scale-invariant, i.e. the relationship $P(k) \sim k^{\lambda}$ is present in any segment of the distribution function. The global and local properties of the function coincide: It is always the same relationship observed through an adequate change in scale. Power-law systems are those where there is no norm, such as exists in a Gaussian distribution. In power-law systems, we can say that indifferentiation exists, a point to which we will return at the end of this article.

It must be emphasised that the invariant final critical state does not emerge from individual acts, independently of other identical acts, but instead from interactions between those acts. As we will show, the mechanism that originates a power law is that the more links a page 
already has (how visible or popular it is), the more it will have (the more popular it will become). On the WWW, «popularity is attractive» (Dorogovtsev, Mendes, 2003, p. 25): Popularity attracts popularity. Links are created according to previously created links; if there are many links that point to a certain node, this must be because that node has received a cumulative number of links over time. This mechanism cannot be based on a random linking of pages. Nor can we start from a fixed set of nodes: It is clear that new web pages are always being created. There has to be an evolutionary process in which there are interactions between nodes. It is an imitation process in which new nodes link to already existing nodes in a function of the number of connections that these previous nodes have. The mechanism that originates a final distribution without a characteristic scale was also proposed by Albert Barabási (1999). The model is as follows:

- Growth: At every time step, a new node is added with $m$ edges that link the new node to nodes already present in the system;

- Preferential attachment: The new nodes connect to an existing node, $i$, with a probability $p$ that depends proportionally (linearly) on the degree $k_{i}$ of node $i$, that is, we have the preference function

$$
p k_{i}=k_{i} / \sum_{j} k_{j}
$$

So, in the model, a new node imitates (proportionally) the previous nodes. The following figure provides an illustration of the process.
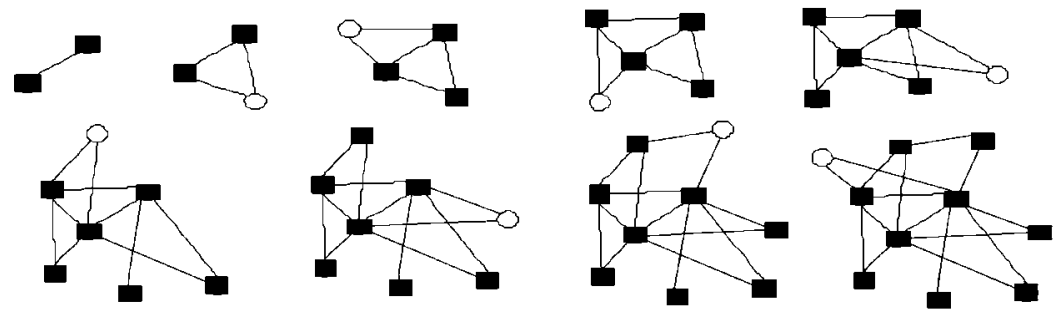

Figure 7. The formation of the space of networks according to the mechanism proposed by Albert Barabási. The new nodes are in white. It is a mechanism of popularity; «popularity is attractive» (Dorogovtsev, Mendes, 2003).

The analytical solution of the model gives the value of the exponent $\lambda=3$ (cf. Barabási et al., 1999). This unique value is not very important here, and in fact, several variations of the model have been constructed that allow us to find a whole spectra of values covering all cases of networks whose empirical values are known. An essential condition of the model is that the preference function must be linear. This point is very important in the argument that we are going to develop, and thus, we underline it. It is equally important to stress again that the model describes a general principle of imitation: New nodes create links by proportionally (linearly) imitating the creation of links by previous nodes.

Let us now analyse more recent types of networks in which imitation is also the main driving force. 


\section{Digital social networks}

After a period when the World Wide Web was the main network based on the Internet, in the middle of the last decade, social networks appeared, such as MySpace, Facebook, Youtube, Twitter, Flickr and Digg, among many others. Originally, these were virtual social networks of «friends». Based upon the definition of a profile, a member invites other «friends» and begins to form a network of links with his «friends». Each «friend» is a node that provides, sends and receives often enormous amounts of content to and from other «friends». In some networks, when the members modify their profiles, their network of friends is automatically notified of such changes, thereby calling attention to themselves..$^{[4]}$ The growth of social networks is rooted in the circular system of causality in which individuals create the content medium, which then reacts upon new individuals, inducing them to join the network, which, in turn, increases the medium's attraction: «friends attract friends». In addition to friendship relationships, the social networks later introduced other forms of social interaction such as «following», «like», «share», «comment», etc. It is clear that most of these forms refer to imitation.

Digital social networks illustrate some of the ideas proposed by René Girard, namely the advance of internal mediation as a characteristic of modernity based on an increasingly intense mimetic process. Traditional mass media such as newspapers, radio or television belonged to an external mediation regime. They were institutionalised organisations, functioning according to norms that regulated how certified professionals produced and disseminated information. They were organisations that controlled what was and when it was transmitted. The information or content was transmitted according to a broadcasting structure one $\rightarrow$ many, in which the «sender» and «receiver» positions were fixed and were clearly differentiated. The «sender» position used a complex and expensive technology with the sole function of «emitting», while the receivers used a simple and inexpensive technology with the sole function of receiving. As has been discussed many times, the receivers were "passive», and the imitation was almost completely vertical: from the model/sender to the passive receivers.

This situation has completely changed in the context of the technological, economic and regulatory factors that have determined the emergence of the new digital media. These factors rely on open networks such as the Internet and the World Wide Web, open networks in the sense that anyone can run programs on these platforms. The other basic technology of the new media is any kind of device that can be called a "computer», which, with some restrictions, is also an open machine (freely programmable) and is present at both the "sender» and «receiver» points of the network. It is the same technology that is present in both positions. It is also a relatively inexpensive technology, allowing anyone to participate in the new open networks, i.e. the Internet, the World Wide Web or social media. Finally, these networks developed with almost no government regulation. These three factors have evolved in tandem with an ever-increasing social mobility in which anyone can aspire to any social position, making the "equality of conditions" that Alexis de Tocqueville identified almost two centuries ago as the main characteristic of modern societies increasingly real. In short, in the new media, particularly in digital social networks, the traditional asymmetric positions of «sender» and «receiver» tend to be undifferentiated, and the communication is increasingly horizontal. Potentially, each individual can, over time, occupy each one of these positions, even if there is asymmetry among the few who communicate much and the many 
who communicate little (power laws, cf. below). The «equality of conditions» or, in René Girard's terminology, the system of internal mediation, has one of its clearest manifestations in the new digital media.

The new interactive media also have a general principle of imitation as a basis for their growth. As René Girard (e.g., Girard, 1987, pp. 292 and sq.), among others, emphasised, imitation can lead to a positive feedback loop. For example, a platform such as Facebook grows through network externalities, which is a form of positive feedback: If an individual joins the networks, this is an incentive for others to imitate him, that is, to adhere as well. It was probably this dynamic that led Facebook, Inc. to program the «friend» relationship as one of the first features of the platform. The «friends» attract new «friends», and it can be demonstrated that the number of new «friends" grows exponentially as a function of the already existing «friends», reinforcing the platform's attractiveness (cf. Shapiro \& Varian, 1998). As mentioned above, there is an imitative process in which the medium is created by the individuals adhering to and publishing content, but this adhesion is also created by the medium which has been created by the individuals who previously joined the network. It is a morphogenetic principle of creation of a medium by individuals who are linked to each other through what they themselves create. It is the circular causality mechanism that is characteristic of positive feedback and in which «cause» (medium) and «effect» (adhesion) merge and are undifferentiated.

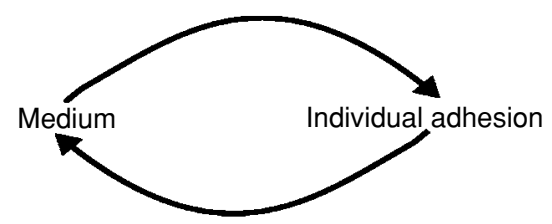

Figure 8. Positive feedback is the growth mechanism of new digital networked media.

It is possible to demonstrate that this dynamic generates monopolies, that is, a platform that is becoming dominant will probably become still more dominant because, as mentioned, the adhesion of new users grows exponentially according to the number of existing users. It is a situation where «the winner-takes-all» (cf. Arthur, 1994), and to which we shall return at the end of this article in a more specifically Girardean framework.

Besides the undifferentiation of positions and network externalities, a more detailed phenomenological analysis of the ways in which individuals interact in social digital networks shows that intersubjective desire, mimetic desire, is present in the intended design of those platforms.

Let us recall that a disciple of René Girard, the founder of PayPal and Imitatio Foundation, Peter Thiel, was one of the first individuals to recognise the ability of a media like Facebook to exploit mimetic desire. In 2004 , he invested $\$ 500,000$ in Facebook because he believed that this network validated Girard's theories: The network is a space of public display designed to attract the desire of others, and it generates cascades of imitation from individual to individual that are analogous to the spread of word of mouth. As Thiel points out, the network is "doubly mimetic».[5] Networks such as Facebook or Instagram are programmed 
to create and intensify the intersubjective desire and the contagion of imitation. The profiles and content associated with each profile (the nodes of the network) are linked by functions mediating desire such as likes, followers, shares and comments. The relationship between Girard's theory and Peter Thiel's investment in a platform of mimetic desire such as Facebook even led someone to say that René Girard was «The Godfather of the like button»! (Sengupta, 2015).

In addition, the desire-manifesting signals conveyed by the links also intensify themselves. As Geoff Shullenberger (2016) pointed out, the more mediated I am through the desire of others, the more attention I receive (measured by likes, for example), so the greater my popularity, the greater the likelihood of attracting new desires («likes») and thus making myself more desirable. Popularity attracts more popularity. Desire begets desire. The new digital networks are spaces displaying individual reciprocal desires. This movement can be thought of as autonomy and differentiation, but the fact is that all individuals are increasingly equal in the universal search for differentiation; all aspire identically or indifferently to the difference manifested in their singularities made public on Facebook or Instagram. The differences oscillate more and more, and they converge towards indifferentiation, since each one can occupy, successively or permanently, the central position of difference that consists in being the object of others' attention. All are potentially, even taking into account the asymmetry in the attention that power law distributions reveal, indifferently the object and the subject of the attention devoted to the public presence of each one in the network. By imitating that public presence, individuals can be simultaneously the models for and the imitators of each other. Communication no longer involves the external mediations existing in traditional media; rather, it is mainly the simple establishment of a social bond between individuals which, of course, merely replicates in digital networks what can be observed in physical social networks. But digital networks are powerful amplifiers of the existing realities. If they permit endless threads of conversation, they are also amplifiers of the intense competition that exists between individuals. This consists of the permanent search for attention, since each individual seeks to be a model for others through the number of «likes» or «followers». This phenomenon has reached an extreme dimension in a network such as Instagram, where winning the competition for the number of followers seems to have become the single purpose for using the network. ${ }^{[6]}$ Always with the help of network theory, the previous phenomenological descriptions can be accurately quantified.

\section{SOCIAL NETWORK METRICS}

As is now clear, interactive digital platforms are networks in a precise sense: Their basic structure always consists of nodes (profiles or content, for example) linked together («friends» or «likes», for example). In these new media, as is also the case on the World Wide Web, popularity is attractive. Since they are networks, the way mimetic desire manifests on those platforms can be accurately quantified. For example, the number of likes, shares and comments on Facebook have a distribution approaching a power law, as shown in Figure 9. In the competition for attention, some individuals are more successful than many others. 


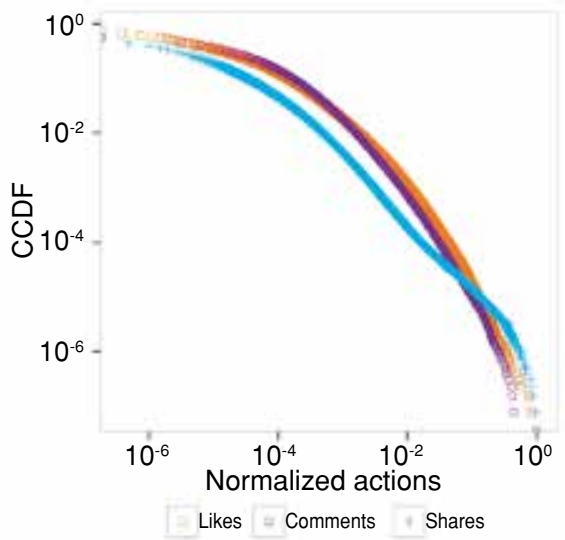

Figure 9. Heavy-tailed distributions on Facebook. Source: Schmidt (2017).

The general metrics of several social networks are well known today. They always attest to power laws and a tendency towards undifferentiation. For example, with respect to the distribution function, one study (Ahn et al., 2007) showed that a major South Korean social network, CyWorld, as well as MySpace, has a power law with the exponent $=2.1$. Another study by Wilson et al. (2009) looked at Facebook (friendship relationships), and also found a power law with the exponent $=1.5$, thus less than the value for the World Wide Web. Another study (Mislove et al., 2008) analysed the social network based on Flickr, the social network present on YouTube and the «pure» social network Orkut, a network that was recently closed. It was shown that the value of the exponent of the distribution function of the inlinks was 1.5., exactly the same value that is observed in the case of the oulinks. The value of the exponent of the outlinks in Flickr is 1.78, while that of the inlinks is 1.74. The value of the exponent of the nodes pointed out on YouTube is 1.99 , while that of the pointing nodes is 1.63. Finally, Twitter displays a distribution function with the exponent $=2.27$ (see table).

These values are very significant and reveal an effective evolution in the dynamics of the massively participative new digital networks. In general, it is confirmed that the values of the exponent of the distribution function in social networks are lower than those of the World Wide Web. More importantly, the exponent values of the outlinks and inlinks are very similar, even with the outlink values being slightly lower than the inlink values. On the contrary, in the case of the WWW, there is a difference between the exponent values of incoming and outgoing hyperlinks: As was mentioned above, $5 \%$ of the nodes receive $75 \%$ of all links, while they only account for $35 \%$ of all links pointing to other pages. In this respect, there is an effective asymmetry on the WWW between sites with incoming links and sites with outgoing links. Conversely, more recent interactive social networks tend to undifferentiate this difference. Some of the cited studies found a high level of symmetry or reciprocity in social networks: If there is a link from friend $A$ to friend $B$, there also tends to be an inverse link. In these networks, if the users receive incoming links, they also tend to link outward to those who link to them, i.e. many network users tend to act reciprocally. The existence of symmetry or reciprocity in links between the users of social networks results in a power law with an exponent that is virtually the same for incoming and outgoing hyperlinks. Nodes with a large number of incoming 
hyperlinks also tend to have a large number of outgoing hyperlinks. There is a positive correlation between incoming and outgoing hyperlinks, an explanation of which is to be found in the positive feedback mechanism, which begets an outgoing link once a user receives an incoming link. There continue to be individuals with more links than others, but now, many who link to others tend to receive links in return, according to a power law. This is the form of the shifts in positions, the trend towards undifferentiation between the «senders» and the «receivers».

Table 1. Values of links distribution (outlinks and inlinks), distance, clustering and reciprocity in the new media.

\begin{tabular}{|l|c|c|c|c|c|}
\hline \multicolumn{1}{|c|}{ Network } & $\begin{array}{c}\text { Exponent } \lambda \\
\text { outlinks }\end{array}$ & $\begin{array}{c}\text { Exponent } \lambda \\
\text { inlinks }\end{array}$ & Distance & Clustering & Reciprocity \\
\hline World Wide Web & 2.7 & 2.1 & 16 & & \\
\hline Cyworld & 2.1. & 2.1. & 7.1 & 0.16 & \\
\hline MySpace & & 2.1. & 2.7 & 0.26 & \\
\hline Orkut & 1.5 & 1.5 & 4.25 & 0.171 & $100 \%$ \\
\hline Yahoo! & 1.5 & 1.7 & & & $84 \%$ \\
\hline Flickr & 1.74 & 1.78 & 5.67 & 0.313 & $62 \%$ \\
\hline YouTube & 1.63 & 1.99 & 5.1 & 0.136 & $79.1 \%$ \\
\hline Facebook & 1.5 & 1.9 & 4.5 & 0.16 & $65 \%$ \\
\hline Twitter & 2.276 & & 4.12 & & $21 \%$ \\
\hline
\end{tabular}

Sources: Broder et al. (2000) for the WWW; Ahn et al. (2007) for Cyworld and MySpace; Mislove et al. (2007) for Orkut, Yahoo! and YouTube; Mislove et al. (2008) for Flickr; Wilson et al. (2009) for Facebook; Kwak et al. (2010) for Twitter.

\section{Power laws AND Mimetic desire}

Participation in the new media is undoubtedly a social practice. As Peter Thiel and Geoff Shullenberger have underlined, digital social networks are programmed to create the conditions for the free propagation of mimetic desire. ${ }^{[7]}$ This is a very important remark about the nature of these networks. On the other hand, we have seen that these networks have a spatial structure (a topology) with very particular characteristics, which is also an important remark. Is it possible to connect these two aspects, i.e. the mimetic desire and the spatial form? This form is the distribution following a power law, in addition to the other metrics mentioned. Is it possible to show that mimetic desire is at the origin of the form of the new media? How can desire be present at the origin of such a precise mathematical function? It should be noted that, now, we are not just repeating that there is a clear manifestation of desire in the structure of the new interactive platforms through «likes», "followers» etc., as was pointed out above. We are rather proposing the hypothesis that mimetic desire generates the spatial form of the new media. It does not seem to be possible for intersubjective desire to generate a spatial 
form. However, we will see that there is indeed a mechanism of social interaction between the individuals participating in the new digital networks that leads them to be connected and to produce content according to a power-law distribution.

In our opinion, the social mechanism of desire that leads to the creation of content in the public space of social networks was definitively identified by Bernardo Huberman and collaborators (2009) in their studies of YouTube, Twitter and a platform that aggregates the links sent by users, Digg. Of course, the authors never use expressions such as «mimetic desire», but it is, in fact, that kind of social interaction that is present in their hypothesis.

As might be expected, Huberman et al. began to find that the number of contributions made by each producer/user of content on these platforms follows a distribution in the form of a power law, as is the case on Digg and YouTube (see Figure 10).
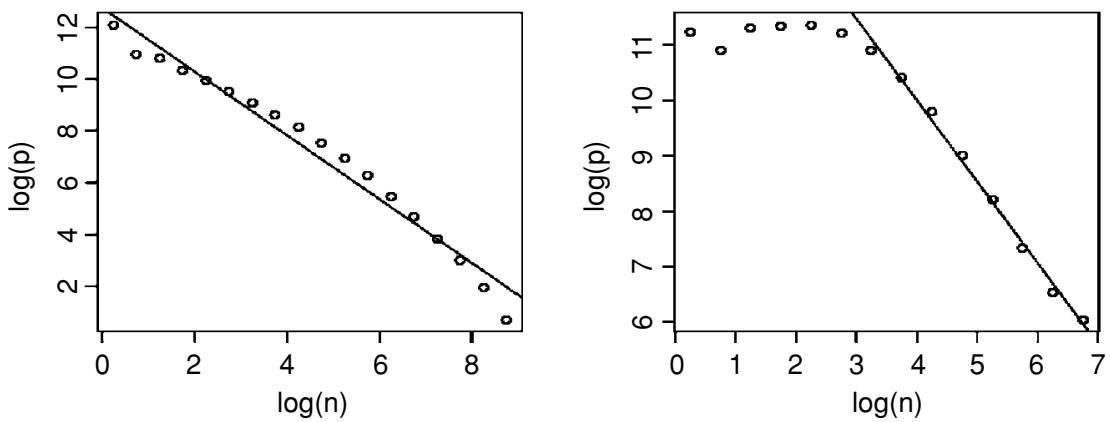

Figure 10. Distribution of the number of contributions for Digg (left) and YouTube (right). The number of contributions follows a power-law distribution on Digg and (with a long tail) on YouTube. Source: Huberman et al. (2009).

More importantly, they found empirically the existence of a positive correlation between the number of content contributions to the platform made by each individual (the productivity) and the popularity (attention measured by the number of «views», «diggs», «likes», «subscribes»), that is, the number of contributions increases with the popularity enjoyed by the content published by each contributor. The authors of the study then proposed the hypothesis that the attention received by each contributor is reinforced over time. What is the explanation for this reinforcement? It consists in a circular link between productivity and popularity or attention. The increasing popularity of an individual increases the attention that he is the object of, which, in turn, increases his productivity, and so on. The more popular the subject, the more he contributes, and more contributions entail more popularity. The mutual reinforcement between (increasing) productivity and (increasing) popularity takes the form of positive feedback.

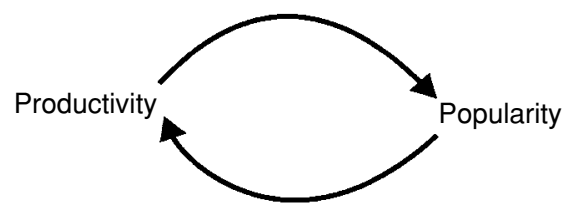

Figure 11. Positive feedback between productivity and popularity. 
To explain why such feedback exists in the first place, the publicity, measured by the number of «fans" (in Digg) and «subscribers» (Youtube), that is, individuals who are automatically notified of the content produced by the contributors, must also be considered. There is again positive feedback. As the authors pointed out, a considerable portion of the attention that a contributor receives can be attributed to her fans. Thus, a contributor with many past contributions (high productivity) naturally has many fans (high publicity). Her fans naturally pay a lot of attention to her next contribution (high popularity). This, in turn, incentivises the contributor to make more contributions (higher productivity), thereby closing the reinforcement loop.

It has also been found empirically (cf. Figure 5 in the aforementioned study) that a contributor's popularity is roughly proportional to her publicity, which, in turn, is roughly proportional to her productivity. Based on the positive feedback mechanism, and given the linear relationship between popularity, publicity and productivity, the authors were able to deduce rigorously the power law distributions which were the starting point. Power laws result from the interactions (measured by attention) between individuals. In our opinion, this is a remarkable result. The form of the media, mathematically characterised, is deduced from a mechanism of social interaction. The form (the topological spatial structure) of the new media, such as the following one is generated by a dynamic which is a kind of mimetic desire! Here, we do not have merely a kinematic model (such as in Barabási's model) but a dynamical one with mimetic desire as the driving force.

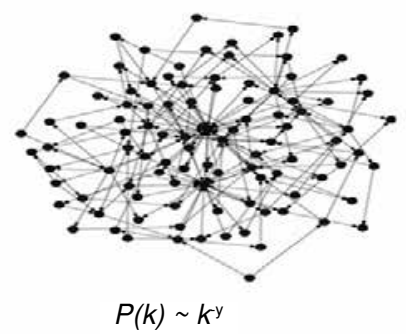

Allow us to clarify this. The deduction of power laws is based on the interactions between contributors and followers. This interaction consists of attention. Attention does not exist, on the one hand, in the contributor or, alternatively, in the follower. It results from the interaction of the two poles of the relationship. By contributing as a function of the attention he receives, the contributor is actually copying this attention, making self-attention the attention that the follower directs towards him. Therefore, his productivity is aimed the continuation of this attention addressed to himself in the future, translating in more self-attention, and thus, fuelling the continuation of his productive effort. It is a form of self-desire, a structure by which the subject's self-desire is a copy of the desire (attention) that others direct towards him, and by which self-desire translates into more productivity and further increases the desire of the others. This corresponds precisely to the structure of the mimetic desire identified by René Girard, more precisely to the type of mimetic desire he called pseudo-narcissism. At the origin of the new media power-law distributions is pseudo-narcissistic desire: The contributor produces because he aspires to the desire of the others. The new public networked media display should definitely be characterised in terms of desire. Mimetic desire theory is indeed 
important to understand the dynamics and formal structure of the new interactive digital media, perhaps even in a deeper way than Peter Thiel and Geoff Shullenberger think.

\section{THE SCAPEGOAT MECHANISM}

We have repeatedly pointed out that a necessary condition for the growth of networks leading to a power-law final distribution is that the number of new connections depends linearly on the existing connections. On the other hand, we have shown that mimetic desire can be the origin of power law distributions such as those found in digital interactive platforms. Now, it is well known that, for René Girard, imitative dynamics is the main force present in a mechanism (the scapegoat mechanism) in which, instead of a few individuals being connected to many, and many to few, all are connected to a single one. That is, all individuals converge to a single individual (the «emissary victim»). In Girard's language, this mechanism occurs when the mimesis of object appropriation turns into a conflictual mimesis, which

will inevitably unify leading two or more individuals to converge on one and the same adversary that all wish to strike down. Acquisitive mimesis is contagious, and if the number of individuals polarized around a single object increases, other members of the community, as not yet implicated, will tend to follow the example of those who are; conflictual mimesis necessarily follows the same course because the same force is involved. (...) Since the power of mimetic attraction multiplies with the number of those polarized, it is inevitable that at one moment the entire community will find itself unified against a single individual (Girard, 1987, p. 26).

Would it be possible to deal with this new situation within the framework of network theory? There must be a final state of polarisation around a single individual caused by a force of imitation that increases with the number of individuals that are already polarised. Network theory formalises the imitative mechanism that constitutes a key in Girard's theory. But it obliges us to distinguish varying degrees in the intensity of imitation, a possibility which, at least explicitly, René Girard does not seem to have contemplated.

To address this new situation, it is important to recall the preferential attachment rule proposed in the context of Barabási's model:

$$
p k i=k i / \sum j k j
$$

That preferential rule can be substituted by the following general attachment rate function:

$$
f(k)=k^{\alpha}
$$

Therefore, the number of $k$ connections or links that a node can acquire depends on the value of $\alpha$, which can be considered a parameter of imitation. As we have seen, imitation can be defined as the number of connections that a node can acquire based on the number of 
connections it already has. But now we are saying that this number varies according to the value of $\alpha$, which is, in fact, a parameter of the intensity of imitation. According to the value of $\alpha$, there are three main possibilities:

- Sublinear case $(\alpha<1)$.

- Linear case $(\alpha=1)$.

- Supralinear case $(\alpha>1)$.

The first possibility is not of much interest: There are some nodes that are more connected than others, but without very noticeable differences in the number of connections. The second possibility, $\alpha=1$, obviously corresponds to the Barabási model. In this case, as we have seen, imitation is also the driving force generating a power law. The third possibility is now the most important. The power of attraction («the power of mimetic attraction», in Girard's words) of a node that initially captures some links grows exponentially. For $1<\alpha<2$, there is a single node that is linked to nearly all nodes. Its power of mimetic attraction indeed multiplies very quickly. In fact, it can further be demonstrated that, for $\alpha>2$, a single node can capture all the links. More accurately, for $\alpha>2$, there is a non-zero probability that a single node links to every other node. ${ }^{[8]}$ The following figure illustrates that result.

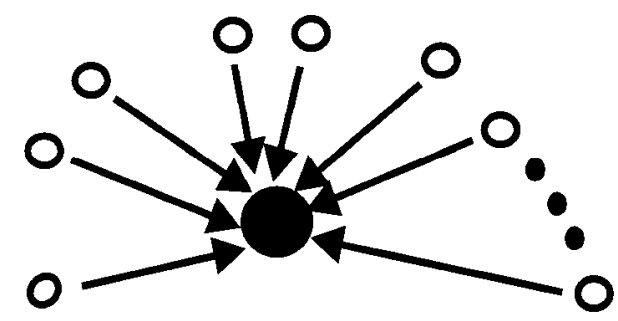

Figure 12. When $\alpha>2$, all nodes are connected to a single node.

Therefore, the mechanism proposed by René Girard corresponds exactly to the situation where $\alpha>2$. It is a case in which the «power of mimetic attraction multiplies». It is a nonlinear dynamic. In fact, an attentive reading of the texts by René Girard could show that, more or less implicitly, they always insist on nonlinear dynamics (see, for example, Girard's insistence on positive feedback). Underlying the dynamics with $\alpha>2$, we have simply tried to clarify the intuition beyond the statement that the power of mimesis «multiplies».

The dynamics $f(k)=k^{\alpha}$, with supralinear $\alpha$, constitute a formal representation of the mechanism in which conflictual mimesis leads all individuals to converge to a single one. It is important to emphasise that, of course, we are not claiming that this representation accounts for the whole of Girard's theory of the origins and development of sacrifice. It represents only the elementary level of that theory. It is a formal process which simply takes account of any situation where, by imitation, all links converge to a single object. For example, as mentioned before, an analogous dynamic exists in the emergence of technological platforms in which «the winner-takes-all», that is, when a monopoly is formed (think of the case of an operating system for personal computers like Windows, for example). A more elaborate formal 
representation of Girard's theory of sacrifice would require other formal models beyond the theory of networks. In particular, the mathematical theory of dynamic systems allows for a deeper analysis of undifferentiating dynamics that, following a power law, converge towards a critical point, ${ }^{[9]}$ which is the kind of dynamics described on certain pages of Violence and the Sacred. ${ }^{[10]}$ Using that theory, one could extend the pioneering analyses conducted by Lucien Scubla on Girard's theory of sacrifice. Even if René Girard himself seems never to have shown much interest in how formal models can help to elucidate the mimetic hypothesis, it is a field of inquiry that shows that his thinking resonates with several recent scientific developments.

\section{References}

Ahn Y., Han,S., Kwak, H., Moon, S., Jeong, H. Analysis of Topological Characteristics of Huge Online Social Networking Service. Proceedings of the Sixteenth International World Wide Web Conference, 835-844. Retrieved from https://www2007.cpsc.ucalgary.ca/ papers/paper676.pdf.

Albert, R., Jeong, H., Barabási, A.-L. (1999). Diameter of the world wide web. Nature, 401, 130-131.

Albert, R., Barabási, A.-L. (2002). Statistical mechanics of complex networks. Reviews of Modern Physics, 74, 47-97.

Arthur W. B. (1994). Increasing returns and Path dependence in the Economy. Ann Arbour: University of Michigan Press.

Bak, P. (1996). How Nature Works. New York: Springer.

Barabási, A.-L., Albert, R., Jeong, H. (1999). Mean-field theory for scale-free random networks. Physica A, 272, 173-187.

Barabási, A.-L. (2002). Linked: The New Science of Networks. Cambridge: Perseus.

Bollabás, B. (1985). Random Graphs. Academic Press: London.

Boyd, D., Ellison, N. (2007). Social network sites: Definition, history, and scholarship. Journal of Computer-Mediated Communication, 13(1), 11.

Broder, A., Kumar, R., Maghoul, F., Raghavan, P., Rajagopalan, Stata, R., Tomkins, A., Wiener, J. (2000). Graph structure in the web. Computer Networks, 33, 309-320.

Dorogovtsev, S. Mendes, J. (2003). Evolution of Networks: From Biological Nets to the Internet and WWW. Oxford: Oxford University Press.

Dupuy, J-P. (1982). Ordres et Désordres - Enquête sur un nouveau paradigme. Paris: Seuil.

Easley, D., Kleinberg J. (2010). Networks, Crowds, and Markets: Reasoning About a Highly Connected World. Cambridge: Cambridge University Press.

Erdös, P., and Renyi, A. (1959). On random graphs. Publicationes Mathematicae, 6, 290-297. Fisher, M. (1983). Scaling, Universality and Renormalization Group Theory. Berlin: Springer.

Girard, R. (1977). The Violence and the Sacred. Baltimore: The John Hopkins University Press.

Girard, R. (1987). Things Hidden Since the Foundation of the World. Stanford: Stanford University Press. 
Huberman, B., Wu, F., Wilkinson, D. (2009). Feeback Loops of Attention in Peer Production. Proceedings of SocialCom-09: The 2009 International Conference on Social Computing. Retrieved from http://www.hpl.hp.com/research/scl/papers/feedbacks/feedbacks.pdf. Krapivsky, P., Redner, S., Ben-Naim, E. (2010). A Kinetic View of Statistical Physics. Cambridge: Cambridge University Press.

Kwak, H., Lee, C., Park, H., Moon, S. (2010). What is Twitter, a Social Network or a News Media? The 19th international conference on World wide web, 591-600.

Mislove, A., Koppula, H., Gummadi, K., Druschel, P., Bhattacharjee, B.(2008). Growth of the Flickr Social Network. Proceedings of the 1st ACM SIGCOMM Workshop on Social Networks (WOSN'08), Seattle, WA.

Mislove, A., Marcon, M., Gummadi, K., Druschel, P., \& Bhattacharjee, B. (2007). Measurement and Analysis of Online Social Networks. Proceedings of the $7^{\text {th }}$ ACM SIGCOMM Conference on internet Measurement, 29-42.

Newman, M.E.J., (2003). The structure and function of complex networks. SIAM Review, 45, 167-256.

Orléan, A. (1985). Monnaie et spéculation mimétique. In Paul Dumouchel (Ed). Violence et Vérité - Autour de René Girard, 147-158. Paris: Grasset.

Schmidt, A., Zollo F., Vicario M,, Bessi A., Scala, A., Caldarelli G., Stanley, H., Quattrociocchi W, (2017) . Anatomy of news consumption on Facebook. Proc Natl Acad Sci U S A, 3035-3039. Scubla, L. (1998). Lire Lévi-Strauss. Paris: Odile Jacob.

Sengupta, A. (2015). The Godfather Of The Like Button Died. Retrieved from https://www. linkedin.com/pulse/godfather-like-button-dead-long-live-his-work-arnaud-auger.

Shapiro, C., Varian, H. (1998). Information Rules. Cambridge: Harvard Business School Press, Cambridge.

Shullenberger, G. (2016). Mimesis, Violence, and Facebook: Peter Thiel's French Connection (Full Essay). Cyborgology. Retrieved from https://thesocietypages.org/ cyborgology/2016/08/13/mimesis-violence-and-facebook-peter-thiels-french-connectionfull-essay.

Watts, D. J. (2003). Six Degrees: The Science of a Connected Age. New York: Norton.

Wilson, C., Boe, B., Sala, A., Puttaswamy, K., \& Zhao, B. (2009). User interactions in social networks and their implications. EuroSys '09 Proceedings of the 4th ACM European conference on Computer systems, 205-218 Retrieved from https://www.cs.ucsb. edu/ ravenben/publications/pdf/interaction-eurosys09.pdf.

\section{ENDNOTES}

[1] In 1980, Jean-Pierre Dupuy organised a colloquium at Stanford, «Disorder and Order», in which several prominent researchers in the area of complex systems participated. It was later published in Dupuy (1982).

[2] Among the many available sources on network theory, Dorogovtsev, Mendes (2003) must be cited at an advanced level, as well as Albert and Barabási (2002) and Newman (2003). At an intermediate level, cf. Easley, Kleinberg (2010). At a more accessible level, cf. the excellent work of Barabási (2002) and Watts (2003). In these references many of the results cited throughout this article can be found. 
[3] More precisely, the clustering coefficient is the ratio of the number of connections between the neighbouring nodes of a given node and the total number of possible connections between those nodes.

[4] Cf. Boyd, Ellison (2007) for an earlier overview of social networking.

[5] Hardy, Q. (2015, November 10). René Girard, French Theorist of the Social Sciences, Dies at 91. The New York Times. Retrieved from https://www.nytimes.com/2015/11/11/arts/international/rene-girard-french-theorist-of-the-social-sciences-dies-at-91.html.

[6] A search on the Internet reveals hundreds of strategies that promise to win the competition for likes in Instagram. More pertinent is the testimony of several women published in The Guardian: Young women on Instagram and self-esteem: «I absolutely feel insecure». (2015, November 4). The Guardian. Retrieved from https://www.theguardian.com/media/2015/nov/04/instagram-young-women-self-esteem-essena-oneill.

[7] Facebook's founding president Sean Parker was also very clear about the intended design of the network from its outset: «it's a social-validation feedback loop ... exactly the kind of thing that a hacker like myself would come up with, because you're exploiting a vulnerability in human psychology.» Ex-Facebook president Sean Parker: Site made to exploit human «vulnerability». (2017, November 9). The Guardian. Retrieved from https://www.theguardian.com/technology/2017/nov/09/facebook-sean-parker-vulnerability-brain-psychology.

[8] A demonstration of that result can be found in Krapivsky et al. (2010, pp. 456-57). Simulations of the network behaviour under a nonlinear dynamics controlled by parameter $\alpha$ can be seen at: https:// www.youtube.com/watch?v=85R_AGXk2Ko.

[9] This is a fundamental result of the theory of critical phenomena in physics, cf. Fischer (1983), and complex systems in general, cf. Bak (1996).

[10] See Girard (1977, p. 158 and sq.), where he describes the path to the sacrificial crisis as when «differences begin to shift back and forth» and «the reciprocal relationships are the sum of nonreciprocal moments». 